\title{
The Natural History of Pancreatitis-Induced Splenic Vein Thrombosis
}

\author{
T. Ryan Heider, MD, * Samreen Azeem, MD, * Joseph A. Galanko, PhD, $†$ and Kevin E. Behrns, MD*†
}

Objective: To determine the natural history of pancreatitis-induced splenic vein thrombosis with particular attention to the risk of gastric variceal hemorrhage.

Summary Background Data: Previous studies have suggested that splenic vein thrombosis results in a high likelihood of gastric variceal bleeding and that splenectomy should be performed to prevent hemorrhage. Recent improvements in cross-sectional imaging have led to the identification of splenic vein thrombosis in patients with minimal symptoms. Our clinical experience suggested that gastric variceal bleeding in these patients was uncommon.

Methods: A computerized index search from 1993 to 2002 for the medical records of patients with a diagnosis of pancreatitis was performed. Fifty-three patients with a diagnosis of pancreatitis and splenic vein thrombosis were identified. The medical records of these patients were reviewed, and follow-up was completed, including the European Organization for Research and Treatment of Cancer Quality of Life Questionnaire (EORTC-QLQ).

Results: Gastrosplenic varices were identified in 41 patients $(77 \%)$ with varices evident on computed tomography (CT) in 40 of 53 patients, on esophagogastroduodenoscopy (EGD) in 11 of 36 patients, and on both CT and EGD in 10 of 36 patients. This risk of variceal bleeding was $5 \%$ for patients with CT-identified varices and $18 \%$ for EGD-identified varices. Overall, only 2 patients $(4 \%)$ had gastric variceal bleeding and required splenectomy. Functional quality of life was better than historical controls surgically treated for chronic pancreatitis.

Conclusion: Gastric variceal bleeding from pancreatitis-induced splenic vein thrombosis occurs in only $4 \%$ of patients; therefore, routine splenectomy is not recommended.

(Ann Surg 2004;239: 876-882)

From the *Department of Surgery, Division of Gastrointestinal Surgery, and Center for Pancreatic and Biliary Disorders, and the †Center for Gastrointestinal Biology and Disease, University of North Carolina at Chapel Hill, Chapel Hill, North Carolina.

Reprints: Kevin E. Behrns, MD, Division of Gastrointestinal Surgery, CB\#7081, 320 Medical Wing E, University of North Carolina at Chapel Hill, Chapel Hill, NC 27599-7081. E-mail: Kevin_Behrns@med.unc.edu. Copyright (C) 2004 by Lippincott Williams \& Wilkins

ISSN: 0003-4932/04/23906-0876

DOI: $10.1097 / 01$. sla.0000128685.74686.1e
$\mathrm{T}$ hough first recognized over 80 years ago as a cause of gastrointestinal hemorrhage, ${ }^{1}$ the natural history of pancreatitis-induced splenic vein thrombosis with subsequent gastric variceal bleeding remains poorly defined. Historically, $45 \%$ to $72 \%$ of patients with splenic vein thrombosis presented with gastric variceal bleeding, ${ }^{2}$ and the overwhelming majority of these patients underwent splenectomy. The reported frequency of splenic vein thrombosis, however, has increased recently due to significant advances in cross-sectional diagnostic imaging. In the remote past, splenic vein thrombosis was recognized only on postmortem examination, ${ }^{1}$ but studies such as celiac angiography and splenoportography have proved useful as diagnostic imaging has evolved. Increasingly sensitive, noninvasive studies such as computed tomography (CT), magnetic resonance imaging (MRI), and ultrasound (US) are currently obtained frequently in the evaluation of patients with acute or chronic pancreatitis, and splenic vein thrombosis is identified. Splenic vein thrombosis complicates pancreatitis or pancreatic pseudocysts in 7 to $20 \%$ of patients, and it is not infrequently diagnosed as patients' symptoms are resolving. ${ }^{3,4}$ Because splenic vein thrombosis is more readily detected with improved imaging and early gastric variceal bleeding is uncommon, an opportunity to document the natural history of this disease has arisen.

Previous studies cited the frequency of gastric variceal hemorrhage as the critical event determining the management, either observation ${ }^{3,5}$ or splenectomy. ${ }^{2,3,6-8}$ An accurate assessment of the incidence of pancreatitis-induced splenic vein thrombosis and the frequency of gastric variceal hemorrhage, however, has been limited by several factors. First, the inclusion of pancreatic malignancies and extrapancreatic etiologies as a cause of splenic vein thrombosis and gastric variceal bleeding confounded the diagnosis and limited our ability to determine the long-term risks. In addition, the lack of a reliable diagnostic imaging modality impaired early diagnosis and follow-up examination. Therefore, our aim was to determine the natural history of pancreatitis-induced splenic vein thrombosis with specific attention to the development of gastric variceal hemorrhage. We found that only $4 \%$ of patients with pancreatitis-induced splenic vein throm- 
bosis developed clinically evident, gastric variceal bleeding during follow-up.

\section{METHODS}

This study was reviewed and approved by the University of North Carolina Chapel Hill Committee on the Protection of the Rights of Human Subjects.

A computerized search for all patients with a diagnosis of acute pancreatitis, chronic pancreatitis, or pancreatic pseudocyst from 1993 to 2002 was performed. Documentation of splenic vein thrombus or venous-phase nonopacification on CT or MRI was considered evidence of splenic vein thrombosis. Fifty-three patients (39 male; mean age, $46 \pm 1.8$ years) with clinical, laboratory, or radiographic evidence of pancreatitis and radiographically-confirmed splenic vein thrombosis were identified. Patients with evidence of cirrhosis, malignancy, or hypercoagulable states were excluded. The medical records were reviewed in detail, and patient demographics, clinical presentation, laboratory data, imaging studies, procedures, and hospital course were recorded. Follow-up data were obtained by standardized telephone interview and administration of the European Organization for Research and Treatment of Cancer Quality of Life Questionnaire (EORTC-QLQ) or from the last recorded clinical evaluation. The EORTC-QLQ has been used previously to assess functional outcome in patients with pancreatic disease, ${ }^{9-11}$ and it permits functional and symptom scale assessment. Functional evaluation includes assessment of physical status, working ability, cognitive function, emotional well-being, social well-being, and global quality of life. Symptom evaluation includes fatigue, nausea and vomiting, pain, loss of appetite, dyspnea, sleep disturbance, constipation, diarrhea, and financial strain. All survey scores were linearly transformed to a 100-point scale for comparative purposes, and higher scores represent improved functional status.

\section{Data Analysis}

Data are reported as the mean \pm SEM, or, where appropriate, median values with the range are shown. Comparisons were made using the Fisher exact test. $P \leq 0.05$ was considered significant.

\section{RESULTS}

\section{Demographics}

Fifty-three patients had splenic vein thrombosis secondary to pancreatitis or the presence of a pseudocyst. The mean age at diagnosis was $46 \pm 1.8$ years, and 39 patients (74\%) were male. Splenic vein thrombosis was due to acute pancreatitis in 13 patients (25\%) and secondary to chronic pancreatitis in the remaining 40 patients (75\%). Pancreatitis was most commonly caused by alcohol use, followed by gallstones, idiopathic pancreatitis, hyperlipidemia, and pancreas divisum (Table 1).

\section{Diagnostic Evaluation}

CT was performed in all patients and demonstrated splenic vein thrombosis in $22(42 \%)$ and splenic vein nonopacification in $31(58 \%)$. Thrombosis was limited to the splenic vein in 34 patients (64\%), but it also involved the superior mesenteric vein, portal vein, or both in $10(19 \%), 3$ $(6 \%)$, and $6(11 \%)$ patients, respectively. In addition to splenic vein thrombosis, CT demonstrated gastrosplenic varices in 40 patients (75\%), and nearly $90 \%$ of all patients had pancreatic pseudocysts (Tables 2 and 3). Ultrasound, MRI, and angiography were performed in addition to CT and confirmed splenic vein thrombosis in $7(13 \%), 6(11 \%)$, and $3(6 \%)$ patients, respectively.

Esophagogastroduodenoscopy (EGD) was performed in $36(68 \%)$ of the 53 patients, and gastric varices were documented in 11 patients (31\%) (Table 3). A single patient had varices on an EGD, but not on CT. Even though varices were present in $75 \%$ of patients undergoing $\mathrm{CT}$, only 2 of these patients $(5 \%)$ had variceal bleeding (Table 3 ). The rate of bleeding was higher in patients who had varices on EGD with $2(18 \%)$ of 11 patients experiencing variceal bleeding. The presence of varices on both CT and EGD did not predict a higher rate of bleeding than EGD alone.

\section{Clinical Course}

Twenty-six patients (49\%) were observed without intervention, 6 patients $(11 \%)$ underwent percutaneous pseudocyst drainage, and $21(40 \%)$ had operative procedures (Table 4). Importantly, intervention was not necessarily temporally or causally related to the diagnosis of splenic vein thrombosis. Five patients in the study population underwent splenectomy, however, only 2 were related to gastric variceal bleeding (Table 4). The first patient developed splenic vein

TABLE 1. Patient Demographics

\begin{tabular}{lc}
\hline & $\mathbf{n}(\mathbf{\%})$ \\
\hline Number of patients & 53 \\
Mean age (yr) & $45.5 \pm 1.8$ \\
Sex (\% male) & $39(73.4)$ \\
Pancreatitis & \\
$\quad$ Acute & $13(25)$ \\
Chronic & $40(75)$ \\
Etiology & \\
Alcohol & $31(58.5)$ \\
Gallstones & $13(25.0)$ \\
Idiopathic & $6(11.3)$ \\
Hyperlipidemia & $2(3.8)$ \\
Pancreas Divisum & $1(1.9)$ \\
\hline
\end{tabular}


TABLE 2. Radiographic Findings

\begin{tabular}{lc}
\hline & $\begin{array}{c}\mathbf{n}(\mathbf{\%}) \\
(\mathbf{n}=\mathbf{5 3})\end{array}$ \\
\hline Splenic vein thrombosis & $22(41.5)$ \\
Splenic vein nonvisualization & $31(58.5)$ \\
Extent of thrombosis & \\
$\quad$ Splenic vein only & $34(64.3)$ \\
Splenic and superior mesenteric vein & $10(18.7)$ \\
Splenic and portal vein & $3(5.7)$ \\
Splenic, superior mesenteric, and portal vein & $6(11.3)$ \\
Pseudocyst & $47(88.7)$ \\
Gastrosplenic varices & $41(77.4)$ \\
\hline
\end{tabular}

thrombosis secondary to chronic alcoholic pancreatitis and required a splenectomy 5 months later for a non-life-threatening gastric variceal bleed. The second patient had splenic and SMV thrombosis secondary to alcoholic pancreatitis and in the face of excessive anticoagulation developed gastric variceal bleeding 2 months after diagnosis. Both patients' varices were documented by EGD and CT and were successfully treated by splenectomy without further episodes of bleeding. The remaining 3 patients underwent splenectomy during operative treatment of pancreatic pseudocysts complicated by splenic parenchymal involvement ( 2 patients) or treatment of a refractory distal pancreatic fistula (1 patient).

Additionally, 3 episodes of nonvariceal upper gastrointestinal hemorrhage caused by a gastric ulcer, gastric cancer, and gastritis occurred in the study population (Table 5). The clinical picture of hypersplenism was noted in only 1 patient, though nearly two thirds of patients manifested abnormal platelet counts with thrombocytopenia and thrombocytosis occurring in $10(19 \%)$ and $22(42 \%)$ patients, respectively. Of the $7(13 \%)$ deaths in the series, none were related to variceal bleeding or the result of an operative complication (Table 5).

\section{Follow-up}

The mean length of follow-up for the entire cohort was $40.3 \pm 4.9$ months (median, 34 months). The mean length of follow-up for the 29 (63\%) of 46 of evaluable patients who were interviewed and completed the EORTC-QLQ survey was $58.5 \pm 3.9$ months. None of the interviewed patients reported further hospitalization or treatment of upper gastrointestinal hemorrhage or reported signs and symptoms consistent with occult intestinal bleeding. Median values for the 6 core functional scales did not differ from previous reports $^{9-11}$ of patients with chronic pancreatitis completing the survey (Table 6). Likewise, symptom scale scores suggest that pancreatitis complicated by splenic vein thrombosis does not result in disabling symptoms; in fact, most patients have few symptoms and function better than historical controls who had surgical treatment of chronic pancreatitis.

\section{DISCUSSION}

Heretofore, prophylactic splenectomy to prevent gastric variceal hemorrhage has been recommended for patients with splenic vein thrombosis, but the benefit of splenectomy was difficult to determine because the natural history of was relatively unknown. Previous studies may have overestimated the frequency of gastric variceal hemorrhage due, in part, to limited diagnostic tools and etiologic heterogeneity. Therefore, our aim was to determine the natural history of pancreatitis-induced splenic vein thrombosis with a specific interest in the frequency of gastric variceal hemorrhage. Our data demonstrate that observation of patients with pancreatitisinduced splenic vein thrombosis is associated with an overall incidence of gastric variceal hemorrhage of approximately $4 \%$, with no deaths secondary to variceal bleeding. Varices demonstrated on CT were associated with a $5 \%$ risk of bleeding, but the presence of varices on endoscopy increased this risk nearly 4 -fold. Of all cases of upper gastrointestinal bleeding in this series, less than half were secondary to bleeding gastric varices, emphasizing the need for a thorough evaluation of bleeding in patients with splenic vein thrombosis. Additionally, this study shows that observation of splenic vein thrombosis does not negatively affect quality of life compared with reported series of patients undergoing surgical treatment of chronic pancreatitis.

TABLE 3. Detection of Varices

\begin{tabular}{lcccc}
\hline Modality & n (\%) & Varices Present (\%) & Variceal Hemorrhage* $^{*}$ & Percent Variceal Hemorrhage \\
\hline Total (any modality) & $53(100)$ & $41(77.4)$ & $2(3.8)$ & $4.9 \%$ \\
CT & $53(100)$ & $40(75.5)$ & $2(3.8)$ & $5.0 \%$ \\
EGD & $36(67.9)$ & $11(30.6)$ & $2(5.6)$ & $18.2 \%^{\dagger}$ \\
Both CT \& EGD & $36(67.9)$ & $10(27.8)$ & $2(5.6)$ & $20 \% \%^{\ddagger}$ \\
\hline
\end{tabular}

\footnotetext{
*No patient without varices on CT or EGD bled.

${ }^{\dagger} P=0.07$ vs. CT;

${ }^{\ddagger} P=0.06$ vs. CT.
} 
TABLE 4. Observation and Interventions

\begin{tabular}{|c|c|}
\hline & $\begin{array}{c}n(\%) \\
(n=53)\end{array}$ \\
\hline Observation & $26(49.1)$ \\
\hline Operative treatment & $21(39.6)$ \\
\hline Necrosectomy & $7(13.2)$ \\
\hline External drainage of pseudocyst & $2(3.8)$ \\
\hline Puestow procedure & $3(5.7)$ \\
\hline Cystenterostomy & $4(7.5)$ \\
\hline Distal pancreatectomy and splenectomy & $3(5.7)$ \\
\hline Splenectomy & $2(3.8)$ \\
\hline Percutaneous pseudocyst drainage & $6(11.3)$ \\
\hline
\end{tabular}

Previous studies have addressed the management of splenic vein thrombosis by characterizing its natural history. Earlier reports relied almost exclusively on celiac angiography or splenoportography for the diagnosis of splenic vein thrombosis, ${ }^{6,9,12-14}$ and the frequency of gastrointestinal bleeding in these studies ${ }^{8,12-14}$ ranged from 37 to $100 \%$. Thus, early data suggested that variceal bleeding was a frequent if not unavoidable sequela of splenic vein thrombosis. A 1985 review by Moosa and $\mathrm{Gadd}^{2}$ found that nearly half of all patients with splenic vein thrombosis, and 70\% of those with gastroesophageal varices, presented with bleeding. Such high rates of bleeding prompted several authors ${ }^{2,6}$ to recommend routine splenectomy. Though clearly demonstrating that potentially fatal complications do occur in patients with splenic vein thrombosis, these studies were limited in recognizing minimally symptomatic patients with splenic vein thrombosis because angiography was not routinely em-

TABLE 5. Complications of Management

\begin{tabular}{ll}
\hline & $\begin{array}{c}\mathbf{n}(\mathbf{\%}) \\
(\mathbf{n}=\mathbf{5 3})\end{array}$ \\
\hline Upper GI bleeding & $5(9.4)$ \\
Gastric varices & $2(3.8)$ \\
Other causes & $3(5.7)$ \\
$\quad$ Gastritis & $1(1.9)$ \\
$\quad$ Gastric cancer & $1(1.9)$ \\
$\quad$ Gastric ulcer & $1(1.9)$ \\
Hypersplenism & $1(1.9)$ \\
Deaths & $7(13.2)$ \\
Sepsis & $3(5.7)$ \\
Substance abuse & $2(3.8)$ \\
Cancer & $1(1.9)$ \\
Myocardial infarction & $1(1.9)$ \\
\hline
\end{tabular}

TABLE 6. Functional Outcome

\begin{tabular}{lcc}
\hline & Median (range) & Historical $^{\mathbf{1 0}}$ \\
\hline Functional scales & & \\
$\quad$ Physical status & $100(0-100)$ & $70(20-100)$ \\
Working ability & $100(0-100)$ & $50(0-100)$ \\
Cognitive & $100(50-100)$ & $66.7(40-100)$ \\
Emotional & $100(8.25-100)$ & $66.7(40-100)$ \\
Social & $100(0-100)$ & $66.7(0-100)$ \\
Global quality of life & $66.6(25-100)$ & $57.1(33.3-100)$ \\
Symptom scales & & \\
Fatigue & $89(44.3-100)$ & $66.7(33.3-100)$ \\
Nausea and vomiting & $100(83.5-100)$ & $100(33.3-100)$ \\
Pain & $83.5(33-100)$ & $100(80-100)$ \\
Loss of appetite & $100(33-100)$ & $100(50-100)$ \\
Dyspnea & $100(67-100)$ & $100(66.7-100)$ \\
Sleep disturbance & $100(0-100)$ & $83.3(33.3-100)$ \\
Constipation & $100(33-100)$ & $66.7(33.3-100)$ \\
Diarrhea & $100(67-100)$ & $100(33.3-100)$ \\
Financial strain & $100(33-100)$ & $100(66.7-100)$ \\
\hline
\end{tabular}

ployed in patients without severe symptoms. Consequently, a disproportionately high percentage of patients presented with variceal bleeding; therefore, nearly all patients underwent splenectomy.

Recent studies have focused on the role of expectant management for patients with asymptomatic splenic vein thrombosis. In their series of 37 patients, Loftus et $\mathrm{al}^{5}$ recommended observation for those with asymptomatic sinistral portal hypertension. Though $54 \%$ of their patients were diagnosed with splenic vein thrombosis by celiac angiography or splenoportography, half were evaluated, at least in part, by CT. Findings from this study highlighted the utility of $\mathrm{CT}$ in splenic vein thrombosis and its important role in the diagnosis and management of pancreatic disease. Despite these diagnostic advances, however, two thirds of their patients presented with variceal bleeding, thereby necessitating splenectomy. Thus only 12 remaining patients were available for evaluation of the natural history of splenic vein thrombosis. Similarly, Sakorafas et $\mathrm{al}^{3}$ studied splenic vein thrombosis diagnosed by CT in 34 patients, of whom only 5 presented with variceal bleeding. Though diagnostic improvements likely explain the decrease in variceal bleeding seen at presentation in this study, only 11 patients did not undergo splenectomy, again limiting the number of patients undergoing nonoperative management of splenic vein thrombosis. No patient in this study initially presented with variceal bleeding, further confirming the sensitivity of CT to detect abnormalities in the splenic vasculature. Also, 26 patients had no operative treatment of pancreatitis, and only $2(4 \%)$ patients 
experienced bleeding from gastric varices, suggesting that, indeed, the risk of bleeding from pancreatitis-induced splenic vein thrombosis is low.

Advances in diagnostic radiology aside, heterogeneity in the disease processes causing splenic vein thrombosis may contribute to the differences between this and previous studies. In acute pancreatitis, splenic vein thrombosis is frequently initiated by local, pro-thrombotic, inflammatory changes in the vascular endothelium, extrinsic splenic vein compression by pseudocysts, relatively low pancreatic perfusion, or later in the course of disease pancreatic fibrosis. Unlike other causes of splenic vein thrombosis, such as malignancy, or hypercoagulable states, the factors responsible for pancreatitis-induced splenic vein thrombosis are often transient. Therefore, the natural history of pancreatitis-induced splenic vein thrombosis may differ from that which results from other etiologies. In the report by Loftus et $\mathrm{al}^{5}{ }^{5}$ only 15 of 37 patients had splenic vein thrombosis secondary to pancreatitis, including only 4 of the 12 who were observed. Sakorafas et $\mathrm{al}^{3}$ presented 34 patients with splenic vein thrombosis secondary to pancreatitis with $50 \%$ of those experiencing gastrointestinal bleeding and two thirds ultimately requiring splenectomy. Importantly, however, those patients were surgically selected and may not adequately represent all patients with pancreatitis-induced splenic vein thrombosis. In our series, only 2 of 40 patients with varices detected by CT and 2 of 11 patients with endoscopically detected varices experienced bleeding. No episodes of bleeding occurred in the absence of varices or in patients with varices on $\mathrm{CT}$ but a normal EGD. Another recent study routinely screening patients with pancreatitis for visceral thrombosis found that only 1 of 6 patients with endoscopically demonstrated gastroesophageal varices bled, supporting our finding that the incidence of variceal bleeding is low. ${ }^{15}$

Improvements in diagnostic radiology have allowed a more complete understanding of the natural history of splenic vein thrombosis in the setting of pancreatitis. Routine splenectomy for pancreatitis-induced splenic vein thrombosis is not indicated given the low incidence of gastric variceal hemorrhage (4\%) and the absence of mortality related to variceal hemorrhage. Patients with endoscopically demonstrated gastric varices had a higher incidence of bleeding $(18 \%)$ and should be educated about the risks of bleeding and the potential need for urgent intervention. Patients with splenic vein thrombosis, particularly with varices seen on CT should undergo regular upper gastrointestinal endoscopy with splenectomy reserved for those who experience bleeding or have an increased risk of hemorrhage. Upper gastrointestinal hemorrhage should be evaluated thoroughly in patients with splenic vein thrombosis because nonvariceal bleeding is as common as bleeding from gastric varices.

\section{REFERENCES}

1. Hirschfeldt H. Die Erkankungen der Milz: Die Hepatolineal Erkankungen. J Springer Verlag Berlin. 1920;384.

2. Moosa A, Gadd M. Isolated splenic vein thrombosis. World J Surg. 1985;9:384-390.

3. Sakorafas G, Sarr M, Farley D, et al. The significance of sinistral portal hypertension complicating chronic pancreatitis. Am J Surg. 2000;179: $129-133$.

4. Mortele K, Mergo P, Taylor H, et al. Splenic and perisplenic involvement in acute pancreatitis: determination of prevalence and morphologic helical CT features. J Comput Assist Tomogr. 2001;25:50-54.

5. Loftus J, Nagorney D, Ilstrup D, et al. Sinistral portal hypertension. Ann Surg. 1993;217:35-40.

6. Bradley E. The natural history of splenic vein thrombosis due to chronic pancreatitis: indications for surgery. Int J Pancreatol. 1987;2:87-92.

7. Weber S, Rikkers L. Splenic vein thrombosis and gastrointestinal bleeding in chronic pancreatitis. World J Surg. 2003;27:1271-1274.

8. Evans G, Yellin A, Weaver F, et al. Sinistral (left-sided) portal hypertension. Am Surg. 1990;56:758-763.

9. Bloechle C, Izbicki J, Knoefel W, et al. Quality of life in chronic pancreatitis-Results after duodenum-preserving resection of the head of the pancreas. Pancreas. 1995;11:77-85.

10. Izbicki J, Bloechle $\mathrm{C}$, Broering $\mathrm{D}$, et al. Extended drainage versus resection in surgery for chronic pancreatitis. Ann Surg. 1998;228:771779.

11. Witzigmann H, Max D, Uhlmann D, et al. Quality of life in chronic pancreatitis: a prospective trial comparing classical Whipple procedure and duodenum-reserving pancreatic head resection. J Gastrointest Surg. 2002;6:173-180.

12. Little A, Moossa A. Gastrointestinal hemorrhage from left-sided portal hypertension. Am J Surg. 1981;141:153-158.

13. Johnston F, Meyers R. Etiologic factors and consequences of splenic vein obstruction. Ann Surg. 1973;177:736-739.

14. Keith R, Mustard R, Saibil E. Gastric variceal bleeding due to occlusion of splenic vein in pancreatic disease. Can J Surg. 1982;25:301-304.

15. Bernades P, Baetz A, Levy P, et al. Splenic and portal venous obstruction in chronic pancreatitis. Dig Dis Sci. 1992;37:340-346.

\section{Discussions}

Dr. William H. Nealon (Galveston, Texas): I would like to congratulate the authors for shedding some light on a subject which is infrequently examined as a complication of pancreatitis. I appreciate the authors providing me the manuscript in a timely fashion. I particularly admire the paper because it confirms my bias.

Dr. Behrns and his colleagues have evaluated 53 patients with sinistral portal hypertension and gastric varices. Two of these patients sustained an episode of variceal bleeding necessitating splenectomy. The existing literature on this subject, some recent and some prior to the ready availability of cross-sectional imaging, have warned of a 40 to $70 \%$ chance of bleeding from this problem. In my experience with more than 300 patients with chronic pancreatitis followed longitudinally, many of whom have had splenic or portal vein thrombosis, I have seen no cases of variceal hemorrhage. One of the 2 patients who bled in this series did so as a complication of excessive anticoagulation.

The study is retrospective, which has well-established flaws. Twenty-five percent had a history of acute pancreatitis, 
and $75 \%$ had chronic pancreatitis. Nearly $90 \%$ had pseudocysts, more than half of which were managed operatively or with percutaneous drainage. Quality-of-life interviews were completed on 29 of 46 surviving patients, and remarkably their quality-of-life levels were superior to standard chronic pancreatitis patients. Patients who had EGD were more likely to bleed.

I have a few questions. I have performed splenectomy for severe left-sided abdominal pain. Were you able to determine that any of these patients had this classic pattern of pain?

I am interested by the preponderance of pseudocysts in your series. We certainly see numerous patients with chronic pancreatitis and splenic vein thrombosis without pseudocyst. Can you comment on this high frequency of pseudocyst?

Did pseudocyst drainage affect the varices in any of these patients?

Why would this group's quality of life be superior to most large series of chronic pancreatitis patients? Is it possible that the patients with acute pancreatitis have skewed the data?

Finally, the death rate of $13 \%$ seems high. Nearly half of deaths were attributable to sepsis. Do you know if any of these were post-splenectomy sepsis? And could this possibly be another deterrent to readily performing splenectomy on these patients?

Dr. Attila Nakeeb (Indianapolis, Indiana): Dr. Behrns and his colleagues from the University of North Carolina nicely characterized the natural history and risks of gastroesophageal hemorrhage in patients with splenic vein thrombosis secondary to pancreatitis. In the Midwest, we also don't see gastroesophageal bleeding secondary to splenic vein thrombosis in our patients with pancreatitis. It is clear with the recent use of cross-sectional imaging, especially CT scanning, in many of our patients with pancreatitis that we are identifying these patients while they are asymptomatic.

In the current study, the authors have identified 53 patients with splenic vein thrombosis and pancreatitis over a 10 -year period. Three quarters of these patients were male and had a diagnosis of chronic pancreatitis, and almost $90 \%$ of the patients had an associated pancreatic pseudocyst. Gastric splenic varices were identified in three quarters of their patients on CT scanning. Of the patients who underwent endoscopy, approximately $30 \%$ had identified varices. In their follow-up at a mean of 40 months, the risk of variceal bleeding was only $5 \%$ in patients with varices identified on $\mathrm{CT}$ and 4 times as great in the patients with varices seen on EGD. Only $4 \%$ of their patients required a splenectomy for bleeding.

I have several questions for the authors. Can you tell us how common splenic vein thrombosis is in your patients with pancreatitis? What is the denominator of your study?
Second, is the etiology of pancreatitis related to the splenic vein thrombosis. Is it more common in your patients with alcoholic pancreatitis, and does continued alcohol abuse have any effect on either quality of life or the bleeding?

Third, as you currently recommend EGD for all patients with splenic vein thrombosis seen on CT scanning, have you used or do you have any experience using betablockers in these patients with varices identified to try to minimize venous hypertension and the risk of bleeding?

Fourth, since almost $40 \%$ of your patients underwent operative intervention, does the presence of splenic vein thrombosis alter your operative management in any way? Are you more likely to perform a resection and splenectomy for a pseudocyst as opposed to doing an enteric drainage in these patients?

Dr. Layton F. Rikkers (Madison, Wisconsin): I would like to congratulate Dr. Behrns and his group on an excellent study, which I think confirms what other past series have shown, namely that splenic vein thrombosis is not frequently complicated by variceal bleeding.

My first question is in regards to the diagnosis itself. Many of the patients were diagnosed with splenic vein thrombosis by not seeing the splenic vein on the CT scan and no collaterals being identified. If these were retrospective examinations of CT scans, I am not certain that all of those patients had splenic vein thrombosis. Usually we like to see the absence of the splenic vein plus some collaterals to make the diagnosis of splenic vein thrombosis.

I have had the opportunity to take care of a large group of patients over a long period of time who have bled from varices from extrahepatic portal hypertension, with splenic vein thrombosis, portal vein thrombosis, superior mesenteric vein thrombosis, or combinations of those entities. Most of these patients had a hypercoagulable syndrome. These patients tend to bleed from varices, whereas those with splenic vein thrombosis or portal vein thrombosis secondary to pancreatitis seem to be less likely to bleed. Do you have any thoughts on this?

The most difficult complication with which these patients can present is small bowel variceal bleeding. This tends to occur in patients with superior mesenteric vein thrombosis. It is virtually impossible to identify the site of bleeding, these patients cannot be shunted, and they are very difficult to manage.

If you are operating in the abdomen in a patient with a known splenic vein thrombosis for some other reason, would you take out the spleen while you were there?

Finally, since thrombosis is a much more life-threatening complication in general than bleeding, is there any role for long-term anticoagulation of these patients? 
Dr. T. Ryan Heider (Chapel Hill, North Carolina): Dr. Nealon asked about the clinical syndrome of left-sided abdominal pain. In this series, we did not see any patients presenting with that clinical syndrome; of all patients that were interviewed, none reported its development.

With regard to the frequency of pseudocysts in the series, nearly $90 \%$ of our patients had pseudocysts, which is higher than the rates of $50 \%$ to $60 \%$ reported in the literature. Many of the pseudocysts in our series were small, clinically insignificant pseudocysts that probably would have been undetected in older series but were included for the sake of data completeness. Although higher than previous reports, this may simply reflect improved detection rather than a true difference between study populations.

With regard to whether the drainage of pseudocysts affects the course of splenic vein thrombosis, I certainly think it could play a role. Although a retrospective study, we observed perhaps 10 to 15 patients who underwent serial $\mathrm{CTs}$; in 3 or 4 patients, there was documented resolution of their splenic vein thrombosis after treatment of their pseudocyst, whether operatively or through percutaneous drainage. Additionally, 1 patient's splenic vein thrombosis resolved simply after the resolution of the pancreatitis. It certainly appears that pseudocysts could be a contributing etiologic factor and that addressing them may encourage the resolution of splenic vein thrombosis.

With regard to sepsis, both patients in our series undergoing splenectomy were alive without complication. Of the cases of sepsis in this series, 1 was due to fulminant necrotizing pancreatitis; the other 2 were unrelated to their pancreatic disease. There were no documented cases of postsplenectomy sepsis, but certainly this is a consideration when deciding whether to perform splenectomy.

Dr. Nakeeb asked several questions, the first regarding the denominator of the study or our incidence of splenic vein thrombosis. We queried 3 different databases, acute pancreatitis, chronic pancreatitis as well as pseudocysts, though there was significant overlap in these databases. Knowing from the literature that the incidence of splenic vein thrombosis would most likely be highest in the pseudocyst group, we queried them first, and out of 250 patients drew most of the cases of splenic vein thrombosis. To make sure we weren't missing a large population of cases of splenic vein thrombosis in the other categories, however, we queried 100 of several thousand entries in the acute and chronic pancreatitis, only picking up 1 or 2 additional cases. The overall denominator was 450 , so roughly a $12 \%$ incidence of splenic vein thrombosis.

In response to your question regarding any potential differences between alcoholic and gallstone pancreatitis, we didn't notice any differences, though that may be just due to small numbers. Nearly $60 \%$ of our patients had alcoholinduced pancreatitis, but we weren't able to discern any differences based on etiology of pancreatitis.

The medical management of their portal hypertension, intraoperatively and postoperatively, is something we have not done. That is an interesting idea that we will likely employ in the future.

With regard to your question regarding splenectomy concomitant with other pancreatic operations, as well as Dr. Rikkers' question regarding performing splenectomy during the course of other abdominal operations, I think we would go ahead and perform a splenectomy if the planned operation included a distal pancreatic resection. But we would not choose to perform an alternate pancreatic operation that we felt was less than ideal solely for the purpose of removing the spleen at the same time. For non-pancreatic abdominal operations, we would not perform a splenectomy.

Dr. Rikkers asked about our diagnosis of splenic vein thrombosis with regard to nonvisualization on CT. We looked exhaustively for secondary evidence of splenic vein thrombosis in these patients. Additionally, we talked with radiologists and felt that if you obtained a good quality CT scan with the appropriate venous contrast phase in the setting of pancreatitis, that there was likely splenic vein thrombosis in these cases. However, the majority of patients with nonvisualization either had varices seen by CT or the suggestion of splenic vein thrombosis by ultrasound, MRI, or another modality. In the absence of secondary findings, however, splenic vein nonvisualization may not be absolutely equivalent as visualized splenic vein thrombosis.

We were fortunate not to see any cases of variceal bleeding through the SMV or IMV circulation into the bowel. We certainly understand from the literature that is a difficult problem; unfortunately, we don't have any advice to offer for that particular complication.

Of the 2 patients who bled in our series, 1 was anticoagulated for cardiac reasons, which exacerbated and perhaps precipitated his variceal bleeding. So I think what we know about the natural history from our data, we would not anticoagulate these patients, but rather treat the underlying pancreatitis and/or pseudocysts and await spontaneous resolution of their splenic vein thrombosis. 